
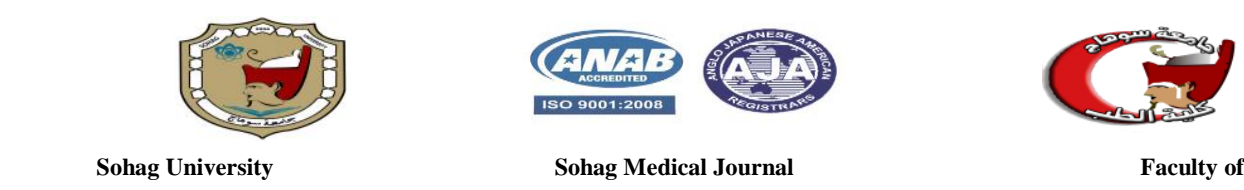

Faculty of Medicine

\title{
Efficacy of diamond microdermabrasion with topical vitamin $C$ in treatment of acne scars
}

\author{
Rasha I. Mohhmed and Marwa A.Aboelmagd \\ Department of Dermatology, Faculty of Medicine, Sohag University
}

\begin{abstract}
:
Introduction: Post acne scars are a common avoidable complication of acne vulgaris. The face is the most common site affected by acne vulgaris and scars so that it has an important impact on the quality of life of these patients.

Treatment of these scars is so important for acne patients but although different treatment methods are available no single treatment has been universally effective. Microdermabrasion (MDA) with infusion is a new modification of MDA technology that includes exfoliation with a dermal infusion.

During this procedure, topical products are introduced into the skin at the time of or immediately after exfoliation.

Aim of the work: Evaluation of efficacy of diamond microdermabrasion with topical vitamin $\mathrm{C}$ in the treatment of acne scars.

Patients and Methods: Ten patients with atrophic post-acne scars in the face attending the outpatient clinic of Dermatology in Sohag University Hospitals between April 2018 to September 2018 were subjected to six diamond microdermabrasion sessions plus administration of topical vit. $\mathrm{C}$ (during the session of diamond microdermabrasion and daily topical application in between sessions). The sessions of diamond microdermabrasion were two weeks apart. patients were evaluated at each session and one month after completion of sessions for both efficacy and safety of the procedure.
\end{abstract}

Results: The mean \pm SD of the patients' age in the study were $26.00 \pm 5$.81years old. Nine females and one male with a mean duration of acne scars \pm SD7.45 \pm 4.73 years, $80 \% \%$ of patients suffered psychological distress from their acne scars. At the end of the study duration, none of the patients achieved grade reduction in their Goodman and Baron qualitative grading score and $100 \%$ of patients had poor $(<25 \%)$ satisfaction.

Conclusion: Multiple sessions of diamond microdermabrasion with topical vitamin $\mathrm{C}$ during sessions and daily application is an ineffective treatment for post-acne atrophic scars

Keywords: diamond microdermabrasion sessions, Vitamin C, acne scar.

\section{Introduction:}

Acne vulgaris is a very common inflammatory skin disease with a high prevalence in adolescents $90 \%$ and persists in $12 \%-14 \%$ of patients into adulthood. It has great psychological and social side effects of ${ }^{(\mathbf{1})}$. Permanent sc- ars of acne resulted from inflammatory acne lesions ${ }^{(2)}$. The prevalence of acne scars in the population is usually in proportion to the severity of acne (3) about $1 \%$ of the population suffer from acne scars ${ }^{(4)}$. 
Acne scars are known to have two types (atrophic and hypertrophic scars) according to the net loss or gain of collagen during the process of healing. Atrophic scars represent eighty to ninety percent of acne scars patients compared to a minority who shows hypertrophic scars and keloids ${ }^{(5)}$. Patients with severe scarring caused by acne are usually suffering from physical and psychological distress, especially in adolescents ${ }^{(2)}$.

Despite the high prevalence of acne scars and multiple treatment methods up till now, there is no single method that is completely effective, each of them has both advantages and disadvantages $^{(\mathbf{6})}$. Microdermabrasion(MDA) with infusion; is a new modification of the technology of MDA that includes exfoliation and dermal infusion. In MDA with infusion, multiple topical treatment agents are introduced into the skin either during or immediately after the exfoliation process. So medications are delivered into the deeper dermal layers $\left.{ }^{(7,8)}\right)$. The beneficial effects of MDA depend on the process of wound healing after the controlled removal of the uppermost layers. This results in the regeneration and cellular renewal of healthier skin ${ }^{(9)}$. So that, several studies have approved findings that support the effective results of microdermabrasion in improving skin contour irregularities, wrinkles, and acne scars ${ }^{(10)}$. The most effective antioxidant in human skin is vitamin $\mathrm{C}$, used newly as a topical treatment for stimulation of collagen formation (11). It leads to effective elevation of the mRNA levels of dermal collagens I and III. Also, vit $\mathrm{C}$ has a role in controlling inhibitors of matrix metalloproteinase 1 in the human dermis (12), and it improves the clinical appearance of photoaged skin and imp-roves facial wrinkles ${ }^{(12)}$.
The application of topical vitamin $\mathrm{C}$ improves the anatomical structure of the dermo-epidermal junction helping the nourishment of the epidermis by increasing the number of nutritive capillary loops in the papillary dermis ${ }^{(13)}$. Energy-based techniques make have multiple side effects and prolonged downtime so non-energy-based techniques like microdermabrasion and topical agents are more suitable to a large number of acne scars patients ${ }^{(6)}$.

Aim of the work: Evaluation of efficacy of diamond microdermabrasion with topical vitamin $\mathrm{C}$ in the treatment of acne scars.

\section{Patients and Methods:}

Design: Cross-sectional clinical study.

Patients: In this study ten patients suffering atrophic facial post-acne scars visiting the outpatient clinic of Dermatology in Sohag University Hospitals between April 2018 to September 2018 were subjected to each patient had six diamond microdermabrasion sessions plus the application of topical Vit. C (during the session and daily topical application in between sessions) diamond microdermabrasion sessions were done two weeks apart. The study had been done according to the ethical guidelines to research humans.

The entire maneuver was described verbally to each patient and the used medication, possible adverse effects, and results. Informed consent was signed from each participant. Patients were evaluated after each session and one month after completion of sessions for both safety and efficacy of the procedure. Complete medical history was taken from each patient and clinical examination was done with evaluation by Goodman and Baron grading syste$\mathrm{m}$ for qualitative description of postacne scar (14) This was done at each session. 
patient self-assessment of the sensation of improvement in scar appearance wa$\mathrm{s}$ recorded as \% of improvement than prior to treatment. Secondary response parameters such as affection of seborrhoea, pore size, and skin texture as reported by patients were also recorded. These parameters were evaluated by direct questioning of the patients after each treatment and one month after the last session in the form of a structured questionnaire in which patients will be asked to rate each parameter as being worse, no change, or improved after the treatment as compared with prior

Goodman and Baron's qualitative evaluation of post-acne scars grades these scars to four grads macular, mild, moderate, and severe.

\section{- The procedure:}

The used device was diamond microdermabrasion.

The treated areas were sterilized with topical alcohol to make degreasing and disinfection effects.

A sterilized diamond probe was used for the treatment The instrument was passed in horizontal, vertical, and oblique directions for 10-20minuts on the treatment area. After treatments, the face was cleaned with sterile normal saline

After each session, immediate application of topical Vit. C serum was done and also once-daily application in between sessions.

The used topical vitamin $\mathrm{C}$ was in the form of a $17 \%$ concentration of L-Ascorbic Acid serum encapsulated in gelatinous capsules each containing $1 \mathrm{mg}$ of the medication. This capsule form for topical use helps to ensure the stability of the agent and also ensure the use of an equal amount in each application

Outcome assessments: At each session and one month after completion of sessions, all patients are evaluated clinically by Goodman and Baron qualitative grading system and Photographic documentation by identical camera setting and lighting was done. Patient satisfaction: was evaluated at the end of the study and was classified as one of four categories that represent the \% of subjective improvement in the shape of acne scars after treatment as compared with before treatment. [Excellent $(100 \%-76 \%)$, Very Good $(51 \%$ $75 \%)$, good $(26 \%-50 \%)$ and poor $(0 \%-25 \%)]$.

Safety assessment: adverse effects and time of recovery were recorded at each treatment session and follow-up visit ( erythema, edema, scaling and crusting, dyschromia, and aggravation of inflammatory acne) before starting session, after each session, and one month after the last session. Inclusion criteria: Facial atrophic acne scars and age greater than 18 years. Exclusion criteria: Active lesions of inflammatory acne, active infection in the area of treatment (e.g., herpes simplex, warts or molluscum), melanoma or lesions suspicious of malignancy, isotretinoin treatment in the past year, dermatoses (e.g., eczema and psoriasis), sunburn, anticoagulant medications and systemic disease (diabetes, hypertension, collagen disease or bleeding tendency).

\section{Results :}

mThe mean \pm SD of the participants" age in the study was $26.00 \pm 5$.81years old. Nine females and one male with a mean duration of acne scars \pm SD $7.45 \pm 4.73$ years, $80 \% \%$ of the patients had psychological distress due to their acne scars. Before starting sessions $50 \%, 40 \%$ and $10 \%$ of patients were grade 2,3 and 4 Goodman and Baron grading system respectively

One month after completion of sessions there was no change in the Goodman and Baron grading system. 
As regard patient self-assessment, $100 \%$ of them report $<25 \%$ improvement ( poor response) while $60 \%$ record improvement in their seborrhea and $50 \%$ report improvement in their skin texture.

\section{Discussion:}

Although a lot of studies were done to evaluate the efficacy of microdermabrasion either alone or in conjunction with other modalities of acne scare therapy, the challenge to compare our results to previous was due to heterogeneity in their sample size, study design, and patient demographics. In addition, different scar grading scales have been used by different studies.

Our study population size was nearly equal to that conducted by Leheta et al. (15), Sharad. (16), El-Domyati et al. (17), Gadkari and Nayak. ${ }^{(18)}$, Leheta et al. ${ }^{(19)}$ to evaluate the efficacy of minimally invasive procedures in treatments of acne scars.

The number of sessions was six sessions one every two weeks near to the average study durations and sessions intervals did by Coimbra et al.(20), Shim et al. ${ }^{(9)}$, and Karimipour et al. (21) For patient evaluation our study combines subjective Goodman and Baron qualitative grading system as used by Majid. (22), Chawla. (23), Nofal et al. ${ }^{\text {(25) }}$, and patient satisfaction that was used by Leheta et al. ${ }^{(19)}$, Nofal et al. (24).

This study is the first study that uses it. $\mathrm{C}$ daily with microdermabrasion session to treat acne scars in a form of Lascorbic acid serum as recommended by studies done by Pinnell et al. (25) and in concentration $17 \%$ that recommended by the study of Matsuda et al. (26) to affect dermal collagen and daily application in between sessions to ensure continuous dermal collagenases.
The only other study that used it. C $15 \%$ with micro-needling was Chawla. (23) that used it. C only during sessions without daily application and compared this with micro-needling with PRP. Although it was found by Coimbra et al. (20) that MDA had a filling effect on fine wrinkles due to the enhancement of new collagen production in the dermis. The same finding was supported on histological and molecular-based studies done by Shim et al. ${ }^{\left({ }^{(9)}\right.}$ and Karimipour et al. ${ }^{(22)}$ respectively. In our study, clinical evaluation of patients by Goodman and Baron grading system shows no improvement in their scars after combining MDA and topical vitamin $\mathrm{C}$.

This can be explained by the need for getting rid of fibrous bands responsible for atrophic scars to be replaced by ne$\mathrm{w}$ collagen formation enhanced by $\mathrm{M}$ DA and topical vitamin C.

\section{Conclusion:}

Multiple sessions of skin microdermabrasion with the application of topical vitamin $\mathrm{C}$ during sessions and daily are not effective in improving post-acne atrophic scars.

\section{References:}

1. Admani S, Barrio VR. Evaluation and treatment of acne from infancy to preadolescence. Dermatologic therapy. 2013;26(6):462-6.

2. Fabbrocini G, Annunziata MC, D'Arco V, De Vita V, Lodi G, Mauriello MC, et al. Acne scars: pathogenesis, classification and treatment. Dermatology research and practice. 2010;2010:893080.

3. Layton AM, Henderson CA, Cunliffe WJ. A clinical evaluation of acne scarring and its incidence. Clinical and experimental dermatology. 1994;19(4):303-8.

4. Cunliffe WJ, Gould DJ. Prevalence of facial acne vulgaris in late adolescence 
and in adults. British medical journal. 1979;1(6171):1109-10.

5. Midwood KS, Williams LV, Schwarzbauer JE. Tissue repair and the dynamics of the extracellular matrix. The international journal of biochemistry \& cell biology. 2004;36(6):1031-7.

6. Kravvas G, Veitch D, Al-Niaimi F. The increasing relevance of biofilms in common dermatological conditions. The Journal of dermatological treatment. 2017:1-6.

7. Lee, Shen, Kuo-Hsien, $\mathrm{Hu} \mathrm{CH}$ and Fang : Lasers and microdermabrasion enhance and control topical delivery of vitamin C. J Invest Dermatol.2003;121:1118-1125

8. Lee, Tsai, Fang, Liu, $\mathrm{Hu} \mathrm{CH}$ and Fag: Micro-dermabrasion as a novel tool to enhance drug delivery via the skin: an animal study. Dermatol Surg.2006; 32:1013-102.

9. Shim, Barnette, Hughes and Greenway: Microdermabrasion: a clinical and histopathologic study. Dermatol Surg.2001;27(6):524-530.

10. Darius Karimipour, Gholamreza Karimipour and Jeffrey Orringer Microdermabrasion: An EvidenceBased Review. Plast. Reconstr. Surg.2010; 125: 372.

11.Oresajo C, Stephens T, Hino PD, Law RM, Yatskayer M, Foltis $P$, et al. Protective effects of a topical antioxidant mixture containing vitamin $\mathrm{C}$, ferulic acid, and phloretin against ultraviolet-induced photodamage in human skin. Journal of cosmetic dermatology. 2008;7(4):290-7.

12. Chen L, Hu JY, Wang SQ. The role of antioxidants in photoprotection: a critical review. Journal of the American Academy of Dermatology. 2012;67(5):1013-24.

13. Sauermann K, Jaspers S, Koop U, Wenck H. Topically applied vitamin C increases the density of dermal papillae in aged human skin. BMC dermatology. 2004;4(1):13.

14.Goodman GJ, Baron JA. Post acne scarring: a qualitative global scarring grading system. Dermatologic surgery: official publication for American Society for Dermatologic Surgery [et al]. 2006;32(12):1458-66.

15.Leheta T, El Tawdy A, Abdel Hay R, Farid S. Percutaneous collagen induction versus full-concentration trichloroacetic acid in the treatment of atrophic acne scars. Dermatologic surgery : official publication for American Society for Dermatologic Surgery [et al]. 2011;37(2):207-16.

16. Sharad J. Combination of microneedling and glycolic acid peels for the treatment of acne scars in dark skin. Journal of cosmetic dermatology. 2011;10(4):317-23.

17.El-Domyati M, Barakat M, Awad S, Medhat W, El-Fakahany H, Farag H. Microneedling Therapy for Atrophic Acne Scars: An Objective Evaluation. The Journal of clinical and aesthetic dermatology. 2015;8(7):36-42.

18.Gadkari R, Nayak C. A split-face comparative study to evaluate the efficacy of combined subcision and dermaroller against combined subcision and cryoroller in treatment of acne scars. Journal of cosmetic dermatology. 2014;13(1):38-43.

19.Leheta TM, Abdel Hay RM, El Garem YF. Deep peeling using phenol versus percutaneous collagen induction combined with trichloroacetic acid $20 \%$ in atrophic post-acne scars; a randomized controlled trial. The Journal of dermatological treatment. 2014;25(2):130-6.

20. Coimbra M, Rohrich RJ, Chao J, Brown SA. A prospective controlled assessment of microdermabrasion for damaged skin and fine rhytides. Plast Reconstr Surg. 2004;113:1438-1443; discussion 1444. 
21. Karimipour DJ, Kang S, Johnson TM, et al. Microdermabrasion:A molecular analysis following a single treatment. $\mathrm{J}$ Am Acad Dermatol. 2005;52:215-223.

22. Majid I. Microneedling therapy in atrophic facial scars: an objective assessment. Journal of cutaneous and aesthetic surgery. 2009;2(1):26-30.

23. Chawla S. Split Face Comparative Study of Microneedling with PRP Versus Microneedling with Vitamin C in Treating Atrophic Post Acne Scars. Journal of cutaneous and aesthetic surgery. 2014;7(4):209-12.

24. Nofal E, Helmy A, Nofal A, Alaska R, Nasr M. Platelet-rich plasma versus CROSS technique with $100 \%$ trichloroacetic acid versus combined skin needling and platelet-rich plasma in the treatment of atrophic acne scars: a comparative study. Dermatologic surgery: official publication for American Society for Dermatologic Surgery [et al]. 2014;40(8):864-73.
25. Pinnell SR, Yang $H$, Omar $M$, Monteiro-Riviere N, DeBuys HV, Walker LC, et al. Topical L-ascorbic acid: percutaneous absorption studies. Dermatologic surgery: official publication for American Society for Dermatologic Surgery [et al]. 2001;27(2):137-42.

26. Matsuda $S$, Shibayama $H$, Hisama $M$, Ohtsuki M, Iwaki M. Inhibitory effects of a novel ascorbic derivative, disodium isostearyl 2-O-L-ascorbyl phosphate on melanogenesis. Chemical \& pharmaceutical bulletin. 2008;56(3):292-7. 